\title{
Modélisation numérique de l'écoulement généré par un déferlement plongeant
}

Stéphane Abadie

Maître de Conférence, Laboratoire de Sciences Appliquées au Génie Civil. ISA-BTP, Université de Pau et des Pays de l'Adour.

Résumé: Une modélisation numérique du déferlement par les équations de Navier-Stokes et un suivi d'interface volumique VOF-CIAM est proposée. La méthode est validée dans le cas de la propagation d'ondes non linéaires permanentes. Le déferlement plongeant sur fond plat d'une onde sinusoïdale de forte cambrure est ensuite étudié. Les caractéristiques du déferlement sont bien traduites par la méthode au moins avant l'impact où des résultats sont déjà publiés. L'intérêt des premiers résultats obtenus après l'impact justifie la démarche et encourage un travail plus systématique à partir de cet outil.

\begin{abstract}
Wave breaking is simulated using the Navier-Stokes equations and the volume tracking algorithm PLIC-VOF. The method is first validated on the case of non linear wave propagation on flat bottom. The plunging motion of a sinusoidal wave of large initial steepness is next studied. The characteristics of the wave up to the impact are found to be well described by the method compared to already publied results. First results obtained after the impact encourage us to go on in this direction.
\end{abstract}

\section{Introduction}

Le déferlement des vagues en zone côtière est un phénomène largement étudié depuis une vingtaine d'années. Ces efforts importants de recherche ont abouti à une meilleure connaissance de l'écoulement avant impact et dans la zone de surf dite « interne » quelques mètres aúdelà de ce dernier. Dans le premier cas, l'écoulement est irrotationnel et le domaine liquide simplement connexe, autorisant le développement de modèles théoriques ou numériques reposant sur l'approximation du fluide parfait (voir par exemple New et al., 1985). Dans le second cas, l'écoulement est quasi-stationnaire (analogie avec un ressaut hydraulique propagatif) et la turbulence bien développée autorisant cette fois l'emploi de modèle statistique de turbulence (voir par exemple Svendsen \& Madsen, 1984). Entre les deux se trouve la zone dite de «transition » marquée par un caractère fortement dynamique, instationnaire et diphasique. La vague y dissipe la majeure partie de son énergie sur les distances très courtes qui la caractérisent. De ce fait, elle revêt une importance fondamentale dans le bilan sédimentaire en zone côtière.

Jusqu'à très récemment pourtant, cet écoulement est resté peu étudié et ce, principalement en raison du manque d'outil mathématique adapté. Une des difficultés est le passage d'un domaine liquide simplement connexe (avant impact) au domaine complexe généré par la reconnexion de l'interface eau/air sur elle-même. Avec l'apparition de nouvelles méthodologies dites de suivi 
d'interfaces motivée par l'étude des problèmes multiphasiques au sens large, ce vide théorique commence lentement à s'estomper. Une seconde barrière, plus difficilement franchissable, est néanmoins liée à la nature transitoire turbulente de l'écoulement. En effet, compte tenu des nombres de Reynolds rédhibitoires d'un tel problème et surtout du caractère non développé de cette turbulence, les outils de description adaptés manquent cruellement et le problème du déferlement est encore loin d'être résolu.

L'étude présentée dans cet article participe de l'effort de recherche vers un essai de caractérisation de cet écoulement complexe. La méthodologie employée repose sur une simulation numérique «directe» des équations de Navier-Stokes diphasiques bidimensionnelles et un suivi d'interface volumique. Après avoir présenté les fondements de cette modélisation et sa validation dans le cas simple de la propagation d'ondes permanentes, les résultats concernant l'écoulement généré par un déferlement plongeant sont analysés.

\section{Présentation de la méthode}

\subsection{Résolution de l'écoúlement}

On considère un domaine fluide semi-périodique horizontalement et composé de deux fluides non miscibles: l'eau et l'air. L'écoulement est supposé incompressible et on néglige en première approximation l'effet de la tension superficielle sur le mouvement. Dans ce cadre, les équations du problème sont simplement les équations de Navier-Stokes incompressibles dans lesquelles la masse volumique et la viscosité sont des fonctions binaires. Il importe donc de connaître à chaque instant la position de l'interface entre les deux fluides afin de pouvoir réinitialiser ces fonctions. Ce sera le rôle du suivi d'interface réalisé de façon indépendante de la résolution de l'écoulement et détaillé dans le paragraphe 2.2. A l'instant initial, la position de l'interface étant connue, le calcul du couple vitesse/pression dans le domaine complet peut être réalisé.

Dans ce but, l'algorithme de projection (Chorin, 1967) suivant a été mis en œuvre:

- un champ de vitesse conservant la quantité de mouvement mais à divergence à priori non nulle est dans un premier temps calculé explicitement comme suit (n étant l'indice temporel courant):

$$
\widetilde{U}=U^{n}+\frac{\Delta t^{n}}{\rho^{n}}\left(\rho^{n} g-\nabla p^{n}+\nabla \cdot \mu^{n}\left(\nabla U^{n}+\nabla^{T} U^{n}\right)-\rho^{n} C\right)
$$

où C est le terme convectif approché par un schéma temporel d'ordre 2 :

$$
C=2 \nabla \cdot\left(U^{n} \otimes U^{n}\right)-\nabla \cdot\left(U^{n+1} \otimes U^{n+1}\right)
$$


- puis l'équation de continuité est résolue par une projection du champ de vitesse intermédiaire sur un espace à divergence nulle :

$$
\mathrm{U}^{\mathrm{n}+1}=\widetilde{U}-\frac{\Delta \mathrm{t}^{\mathrm{n}}}{\rho^{\mathrm{n}}} \nabla\left(\mathrm{p}^{\mathrm{n}+1}-\mathrm{p}^{\mathrm{n}}\right)
$$

Cette étape requiert le calcul implicite du champ de pression par l'équation de Poisson suivante:

$$
\nabla \cdot\left(\frac{\Delta t^{n}}{\rho^{n}} \nabla\left(p^{n+1}-p^{n}\right)\right)=\nabla \cdot \widetilde{U}
$$

Ces équations sont discrétisées sur une grille décalée vitesse/pression suivant la méthode des volumes finis. Le terme convectif est approché par un schéma centré d'ordre 2.

Les conditions aux limites utilisées sont les suivantes : périodicité en vitesse et pression sur les limites latérales, condition de glissement pour la vitesse et Neumann homogène pour la pression sur les limites horizontales. Ces conditions conduisent (pour l'équation (4)) à un système linéaire à 7 diagonales inversé suivant l'algorithme Bi-CGSTAB (Van der Vorst, 1992).

\subsection{Le suivi d'interface}

La méthode employée pour décrire l'évolution temporelle de l'interface eau/air appartient aux méthodes de type VOF (Volume de fluide). L'interface y est décrite par une fonction $F$ traduisant le coefficient de remplissage de l'eau dans chaque maille d'un maillage fixe. Cette représentation permet de traiter facilement le problème de reconnexion de l'interface sur elle-même en comparaison des méthodes à traceurs surfaciques.

L'algorithme VOF-CIAM (Li, 1995) qui a été programmé se décompose de la façon suivante :

- la première étape consiste à reconstruire une interface approchée sous forme de segments linéaires à partir des valeurs de $F$ dans chaque maille.

- Ces segments sont ensuite advectés par l'écoulement (déplacement Lagrangien dans le maillage).

- Enfin, la fonction $F$ est réinitialisée par un simple calcul d'aire.

Le transport Lagrangien permet d'obtenir une précision supérieures aux méthodes VOF antérieures utilisant un calcul de flux pour l'étape d'advection. En effet, ce calcul de flux entraîne généralement une perte d'information par diffusion numérique qui n'apparaît pas ici. 


\section{Validation}

\subsection{Ondes permanentes}

La méthode est dans un premier temps testée dans le cas de la propagation d'ondes permanentes. Des solutions de références sont obtenues par la méthode de Rienecker \& Fenton (1981) consistant à approcher la fonction courant solution du problème potentiel par une série de Fourier. Les simulations réalisées correspondent à des ondes non linéaires. Les résultats de cette validation, présentés dans Abadie et al., 1998, montrent une très bonne corrélation entre la surface libre initiale issue de l'approximation de Fourier et l'interface calculée par VOF après une période de propagation. L'erreur cumulée sur les deux composantes de la vitesse est également en moyenne, dans tout le domaine, de l'ordre de $4 \%$. Ces résultats indiquent un comportement très satisfaisant de la méthode sur l'ordre de grandeur temporel d'une période de vague qui est l'intervalle dans lequel toutes les simulations de l'étude sont restreintes.

\subsection{Déferlement avant impact}

Le déferlement étudié dans cet article est créé par l'instabilité d'une onde sinusoïdale de très forte cambrure initiale sur fond plat. Ce déferlement particulier, dont les caractéristiques sont proches d'un déferlement par remontée de fond, permet de restreindre le nombre de paramètres influençant l'écoulement au nombre de 3 : la cambrure initiale $\mathrm{H}_{0} / \mathrm{L}_{0}$, le rapport profondeur/longueur d'onde $\mathrm{d} / \mathrm{L}_{0}$ et le nombre de Reynolds $\rho \mathrm{cL} / \mu$, le nombre de Froude et le rapport des caractéristiques des deux fluides (viscosité et densité) étant conservés constants par ailleurs.

Le cas étudié dans cet article correspond aux données du tableau 1.

\begin{tabular}{|c|c|c|c|c|c|}
\hline Maillage & $\mathrm{Re}$ & $\mathrm{H}_{0} / \mathrm{L}_{0}$ & $\mathrm{~d} / \mathrm{L}_{0}$ & $\Delta \mathrm{t} / \mathrm{T}$ & $\Delta \mathrm{x} / \mathrm{L}=\Delta \mathrm{y} / \mathrm{L}$ \\
\hline $360 \times 120$ & $10^{4}$ & 0.13 & 0.13 & $5 \times 10^{-4}$ & $2.77 \times 10^{-3}$ \\
\hline
\end{tabular}

Tableau 1 : données de la simulation

Table 1 : simulation data

Ce cas correspond à un déferlement plongeant. On montre (Abadie, 1998) qu'il est possible de générer un ensemble continu de déferlement allant du déversant au très fortement plongeant en jouant sur la cambrure initiale. Le nombre de Reynolds pris dans cette simulation ne correspond évidemment pas aux nombres rencontrés dans le phénomène réel. Du point de vue numérique, il apparaît nécessaire de limiter ce nombre à des valeurs raisonnables afin de pouvoir conduire les calculs. Du point de vue physique, les caractéristiques de l'écoulement dans les premiers instants succédant l'impact (structures tourbillonnaires grande échelle) conduisent à penser que cette viscosité artificielle ne perturbe pas l'interprétation des résultats tant qu'elle reste inférieure à une certaine valeur. 
Le champ des vitesses obtenu dans ce cas par la méthode VOF est comparé (Figure1) à une étude antérieure (Vinje \& Brevig, 1981) menée en fluide parfait par éléments frontières. La corrélation entre les deux résultats est très bonne à la fois sur la position de l'interface et sur la direction et l'intensité des vitesses validant par là même la méthodologie complète. Lors de la phase d'éjection, la méthode VOF permet également de retrouver le résultat déjà connu d'un jet approximativement en chute libre.

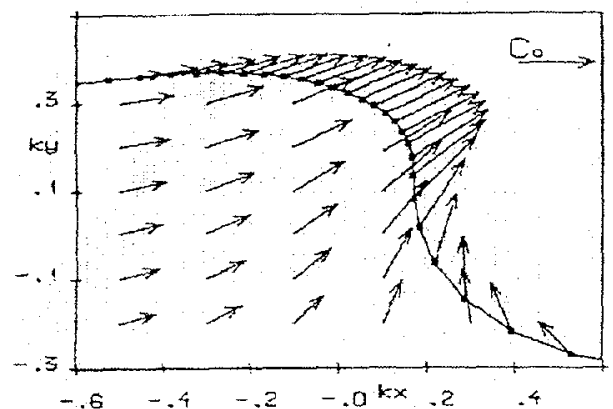

a)

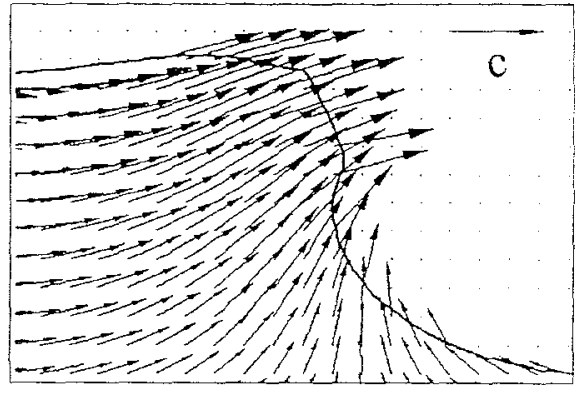

b)

Figure 1: Champ des vitesses, $\mathrm{t}=0.28 \mathrm{~T}$, a) Vinje \& Brevig 1981, b) cette étude Figure 1: Velocity field, $t=0.28 T$, a) Vinje \& Brevig 1981, b) this study

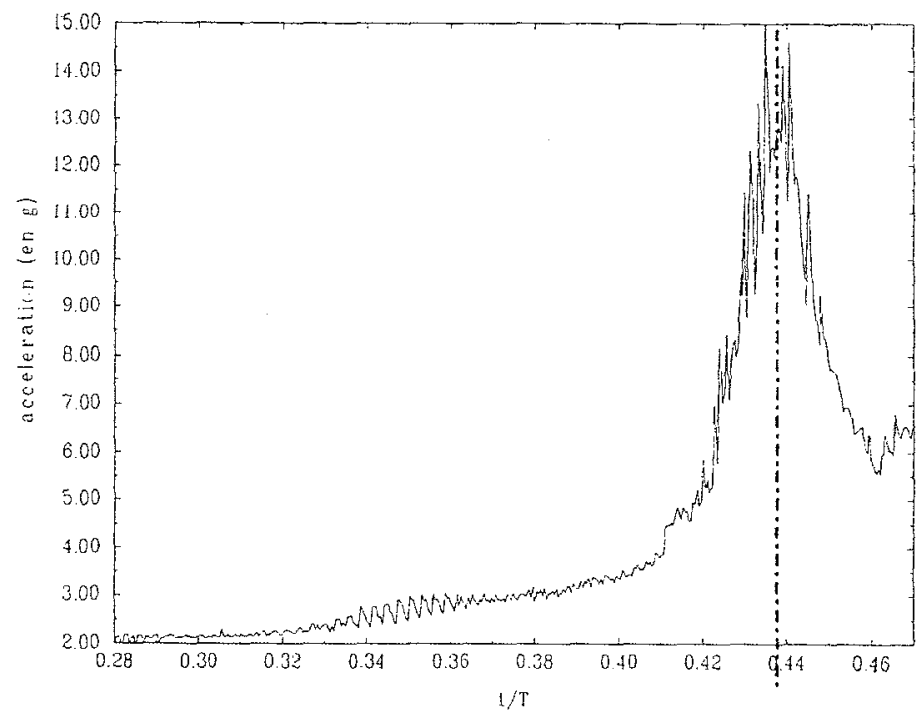

Figure 2: évolution temporelle de l'accélération maximum dans la vague

Figure 2 : time evolution of the maximum of acceleration in the wave.

Différentes études antérieures (par exemple New et al., 1985) montrent que l'accélération est maximum au niveau de la partie verticale inférieure de la vague et peut atteindre 5 à $6 \mathrm{~g}$ juste avant l'impact. Dans la Figure 2 retraçant l'évolution temporelle de ce maximum d'accélération, ce résultat est également retrouvé par la 
méthode VOF avant l'impact. Cependant, une brusque augmentation de ce paramètre (jusqu'à $13 \mathrm{~g}$ dans notre cas) est observé dans les tous derniers instants précédant l'impact. Si ce résultat nouveau mérite confirmation par une étude systématique, la différence entre les deux valeurs pourrait s'expliquer par le fait que la méthode VOF permet d'aller plus loin en temps dans la simulation et donc de décrire une phase inaccessible aux éléments frontières.

\section{Ecoulement après impact :}

L'écoulement post déferlement se caractérise dans un premier temps par l'apparition d'un jet secondaire fortement ascendant. L'origine de ce jet en terme de particules fluides est déterminée dans Abadie et al, 1998. Le jet ascendant apparaît essentiellement constitué de particules initialement au repos (voir Figure 3 ) et non dans le jet déferlant. Un autre résultat est que le jet de déferlement ne pénètre pas la colonne d'eau alors que le déferlement est fortement plongeant. Les particules issues de ce jet sont rapiủement entrainées dans un mouvement circulaire autour de l'air emprisonné.

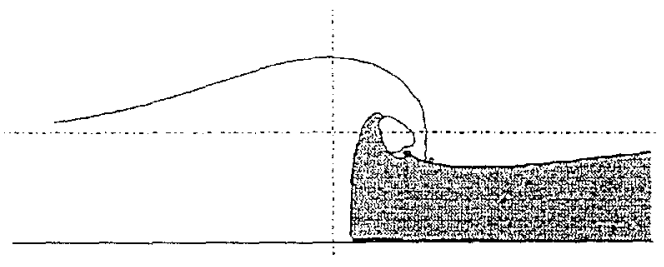

$t=t_{0}$

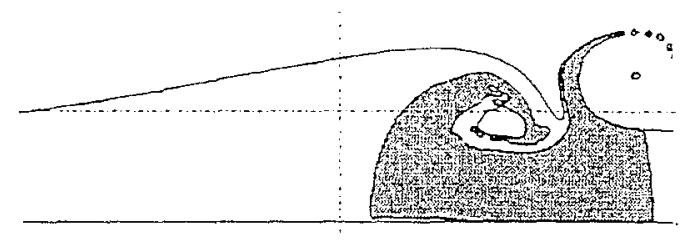

$t=t_{0}+0.11 T$

Figure 3 : Suivi d'un volume d'eau (en gris) an cours du mouvement.

Figure 3 : Tracking of a marked water volume (in grey) during the breaking motion.

Cette zone de circulation est en fait le siège d'une vorticité négative importante qui se crée dès la reconnexion de l'interface et qui entoure le volume d'air piégé. Ce large tourbillon très énergétique acquiert par la suite sa dynamique propre. En effet, il se détache rapidement du mouvement ondulatoire et descend vers le fond y générant de fortes vitesses horizontales (cf. Figure 4). Quantitativement, ces vitesses atteignent alors un maximum de $2 c$ (célérité de l'onde linéaire à cette profondeur) qui est approximativement égale à la vitesse maximum dans le jet déferlant. Ce mécanisme de descente tourbillonnaire pourrait être un des processus principaux de mise en suspension du sédiment lors du déferlement plongeant. 


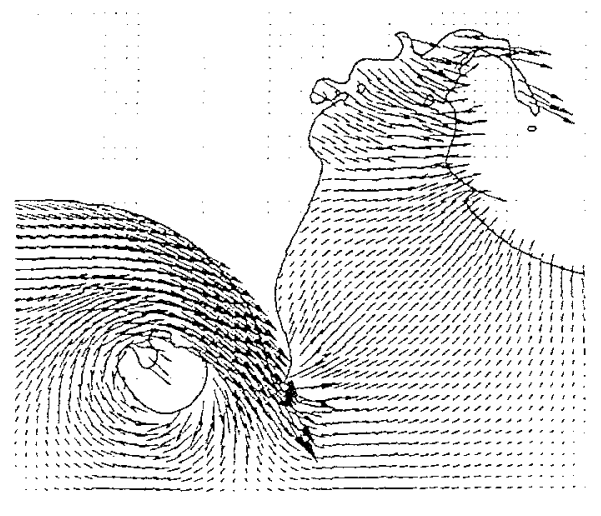

a)

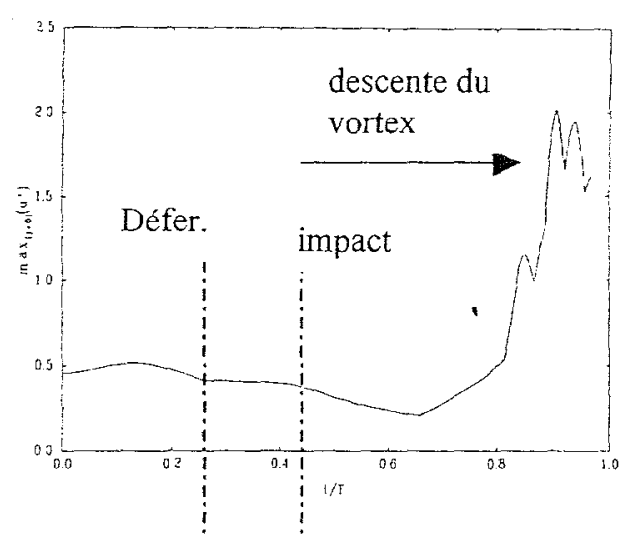

b)

Figure $4:$ a) champ de vitesse à $t=0.78 \mathrm{~T}$

b) Evolution temporelle du maximum de vitesse $u^{*}=u / c$ sur le fond.

Figure 4: a) velocity field at $t=0.78 \mathrm{~T}$

b) time evolution of the velocity maximum on the seabed.

Le principal mécanisme de génération de vorticité est la reconnexion d'interface qui peut intervenir plusieurs fois au cours du mouvement. La première source de vorticité est l'impact du jet déferlant. L'interaction de la crête avec le jet secondaire ascendant en est une seconde (voir Figure 5). L'intensité tourbillonnaire (enstrophie) augmente à chacun de ces évènements d'un ordre de grandeur. Ce phénomène joue sans aucun doute un rôle important dans la dissipation énergétique de la vague.

Comme on pouvait s'y attendre, les pressions calculées apparaissent maximales au point d'impact. On trouve ainsi en variable adimensionnelle $\left(\mathrm{p}^{*}=\mathrm{p} / \rho \mathrm{c}^{2}\right)$ des valeurs de l'ordre de 3 ou 4 correspondant dans ce cas à 3 ou 4 fois la pression hydrostatique. La pression sur le fond reste inchangée jusqu'à la descente du vortex grande échelle qui triple alors cette valeur hydrostatique.

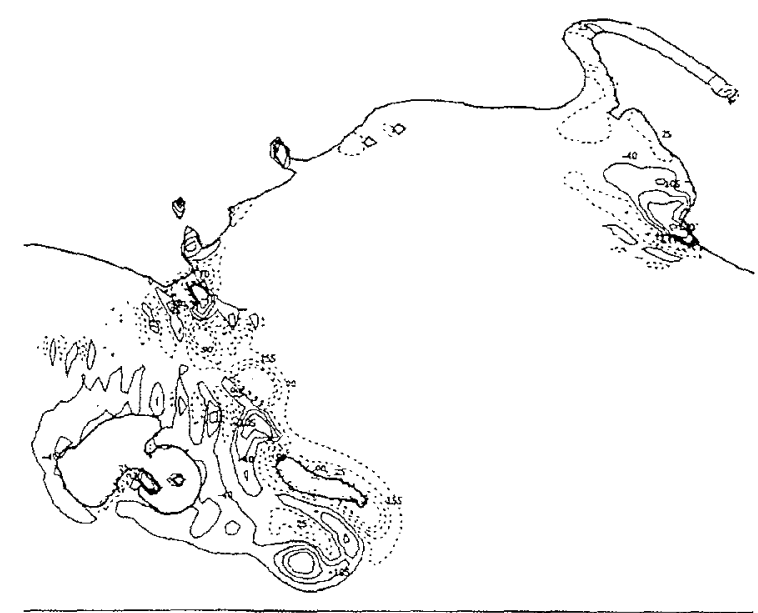

Eigure 5: : Vorticité (adimensionnelle), (trait continu = vorticité négative), Figure 5: : non dimensional vorticity (continu line $=$ negative vorticity)

$$
\omega^{*}=\mathrm{T} \operatorname{rot} \mathbf{U}, \Delta \omega^{*}=65, \omega_{\min }^{*}=-235, \omega_{\max }^{*}=220, \mathrm{t}=0.84 \mathrm{~T}
$$




\section{Conclusions et perspectives :}

La simulation numérique par les équations de Navier-Stokes et le suivi d'interface VOF-CIAM est un outil efficace qui peut permettre à terme une connaissance plus fine de l'écoulement généré par un déferlement. Cette étude se limite volontairement à une configuration simpliste de déferlement sur fond plat afin de limiter également les paramètres d'écoulement. Les premiers résultats actuels nous conduisent à envisager dans un avenir proche une étude paramétrique fonction de ces mêmes paramètres, le but étant d'aboutir à une caractérisation de la zone de transition.

\section{Références bibliographiques:}

Abadie S. M., 1998, Modélisation numérique du déferlement plongeant par méthode VOF, Thèse de Doctorat de Mécanique, Universite Bordeatix I, 16 Janvier.

Abadie S., Caltagirone J. P. et Watremez P., 1998, Mécanisme de génération du jet secondaire ascendant dans un déferiement plongeant, C. R. Ačad. Sci. Paris, t. 326, Série II b, p. 553-559.

Chorin A. J., 1967, A numerical method for solving incompressible viscous flow problem, J. Comp. Phys., 2, 12-26́.

Guignard S., Grilli S. T., Marcer R. \& Rey V., 1999, Computation of Shoaling and breaking waves in nearshore areas by the coupling of BEM and VOF methods, Proc. $9^{\text {th }}$ Intl. Offshore and Polar Engng. Conf. (ISOPE99, Brest, France, mai 1999), III, 304-309. Li J., 1995, Calcul dinterface affine par morceaux (Piecewise Linear Interface Calculation). C. R. Acad. Sci. Paris, Série IIb, Paris, 320, 391-396.

New A. L., Mc Iver P., Peregrine D. H., 1985, Computations of overturning waves, $J$. Fluid Mech., 150, 233-251.

Rienecker M. M., Fenton J. D., 1981, A Fourier approximation method for steady water waves, J. Fluid Mech., 104, 119-137.

Svendsen I.A., Madsen P.A., 1984, A turbulent bore on a beach, J. Fluid Mech., 148, $73-$ 96.

Van der Vorst H. A., 1992, BI-CGSTAB : A fast and smoothly converging variant of BICG for the solution of nonsymmetric linear systems, SIAMJ. Sci. Stat. Comput., 13, 631644.

Vinje T., Brevig P.,1981, Numerical simulation of breaking waves, $J$. Adv. Water

Resour., 4, 77-82. 